\title{
Contribution of multiple inert gas elimination technique to pulmonary medicine
}

Series editor: $R$ Rodriguez-Roisin

\section{Introduction}

\author{
R Rodriguez-Roisin
}

Amongst the four traditional causes of arterial hypoxaemia (alveolar hypoventilation, limitation of alveolar to end capillary oxygen diffusion, intrapulmonary shunting, and ventilationperfusion ( $\left.\dot{\mathrm{V}}_{\mathrm{A}} / \dot{\mathrm{Q}}\right)$ mismatch) and those of hypercapnia (alveolar hypoventilation and $\dot{\mathrm{V}}_{\mathrm{A}} / \mathbf{Q}$ heterogeneity), $\dot{\mathrm{V}}_{\mathrm{A}} / \dot{Q}$ inequality emerges as the most prominent mechanism of the pathophysiology of pulmonary gas exchange in lung diseases. Consequently, the most rational approach to understanding the underlying pathogenetic mechanisms and the therapeutic strategy of arterial blood gas abnormalities should be based on the ability to assess the extent of $\dot{V} A / Q \dot{Q}$ abnormalities.

Historically the assessment of $\dot{V}_{A} / Q$ inequalities has been restricted to the view of the simple three compartmental model of Riley and Cournand $^{1}$ : the ideal lung where ventilation and blood flow are appropriately apportioned; the shunt fraction in which the compartment remains perfused but not ventilated; and the physiological dead space in which lung units are ventilated but not perfused. One of the major disadvantages of these indices is that they are non-specific in the presence of gas exchange abnormalities. For instance, any variation in $\dot{V} A / Q$ relations will induce changes in them; it is also known that both variables are sensitive to changes in overall ventilation and cardiac output. Another problem of paramount importance is that all these simple (respiratory blood gases) or relatively simple (derived indices) measurements of abnormal gas exchange reflect not only the status of the lung and thereby its intrapulmonary determinants - namely, limitation of diffusion of oxygen; shunt; and $\dot{V}_{A} / \dot{Q}$ mismatching - but also the conditions under which the lung is operating - that is, the composition of inspired gas and that of mixed venous blood.

These classical indices of gas exchange were superseded in the early 1970 s by a major conceptual breakthrough - the multiple inert gas elimination technique (MIGET) - based on the principles of mass balance and steady state conditions of inert gas exchange. ${ }^{23}$ Instead of relying upon the traditional respiratory gases MIGET is based on the gas exchange behaviour of six foreign gases and provides a quantum leap forward in our understanding of pulmonary gas exchange. Wagner and associates $^{23}$ recognised that the utilisation of several inert gases of appropriate solubilities improved the resolution at the ends of the spectrum of $\dot{V} A / Q$ relationships much better than in the model of Riley and Cournand. ${ }^{1}$ There are two principal advantages of MIGET: (1) it estimates the patterns of alveolar ventilation and pulmonary blood flow without disturbing either vascular or bronchomotor tone; and (2) it facilitates the unravelling of the extrapulmonary determinants (overall ventilation, cardiac output, and oxygen consumption) of abnormal gas exchange.

With the use of MIGET numerous experimental and clinical contributions have been made over the last two decades. ${ }^{45}$ This series of articles on inert gas studies highlights some of the most relevant clinical and pathophysiological aspects related to acute and chronic pulmonary diseases, an area in which European investigators have been working extensively. The series begins with a review of the principles and physiological concepts of inert gas exchange with the exceptional contribution of one of the original designers of MIGET. A full description of the methodological details of MIGET is provided, a piece of information lacking in the current literature, followed by the characteristics of $\dot{V}_{A} / Q$ relationships in normal man, at rest, and during heavy exercise whilst breathing room air or hypoxic mixtures. From a clinical viewpoint these data are of considerable interest to understanding more clearly gas exchange abnormalities in disease states. The next two articles are devoted to the commonest chronic pulmonary conditions - namely, chronic obstructive pulmonary disease (and cryptogenic fibrosing alveolitis), and bronchial asthma. In patients with chronic expiratory airflow limitation the basic mechanisms of arterial blood gas abnormalities and their potential correlations with structure, the influence of oxygen breathing, and the effects of some of the drugs commonly given are reviewed. Moreover, in patients with lung fibrosis the importance of the pulmonary circulation on gas exchange and the role of alveolar to end capillary diffusion limitation to oxygen at rest and during exercise are highlighted. Likewise, in bronchial asthma the interplay between intrapulmonary and extrapulmonary factors modulating arterial blood gases and the intriguing dissociation between spirometry and gas exchange are emphasised. The fourth article reports on the most striking aspects of pulmonary vascular disorders on gas exchange in which the impact of the key role 
played by cardiac output and mixed venous blood $\mathrm{PO}_{2}$ is conspicuously present. The commonest causes of acute lung injury, adult respiratory distress syndrome, and pneumonia, and several therapeutical strategies are reviewed in the fifth article. Finally, the last article of the series summarises the effect of anaesthesia on pulmonary gas exchange in patients, with and without pulmonary problems, and provides unique information on the elegant correlations between lung imaging (assessed by computed tomography) and gas exchange.

In summary, the purpose of the present series is to provide a solid framework to assist the clinician in the proper interpretation of the complex interplay between the different deter- minants governing arterial blood gases. This is one of the fields in which European respiratory medicine may make a unique contribution to the analysis of clinical problems within pulmonary gas exchange.

1 Riley RL, Cournand A. "Ideal" alveolar air and the analysis of ventilation-perfusion relationships in the lung. $\mathrm{F}$ Appl Physiol 1949;1:825-47.

2 Wagner PD, Saltzman HA, West JB. Measurements of continuous distributions of ventilation-perfusion ratios: theory. f Appl Physiol 1974;36:588-99.

3 Evans JW, Wagner PD. Limits on $\dot{V}_{A} / Q$ distributions from analysis of experimental inert gas elimination. $\mathcal{f} A p p l$ Physiol 1977;42:889-98.

4 Rodriguez-Roisin R, Wagner PD. Clinical relevance of ventilation-perfusion inequality determined by inert gas elimination. Eur Respir $\mathcal{F}$ 1989;3:469-82.

5 Wagner PD, Rodriguez-Roisin R. Clinical advances in pulmonary gas exchange. Am Rev Respir Dis 1991;143:883-8. 